\title{
Uncovering the human evaluation of changeability for automated factory layout planning: an expert survey
}

\author{
Peter Burggräf ${ }^{1} \cdot$ Matthias Dannapfel $^{1} \cdot$ Viviane Hahn $^{1}$ (D) $\cdot$ Michael Preutenborbeck $^{1}$
}

Received: 18 November 2020 / Accepted: 22 December 2020 / Published online: 23 January 2021

(c) The Author(s) 2021

\begin{abstract}
On the way to automated layout planning numerous challenges must be faced. One of these challenges is the automated layout evaluation, which can be considered as the core of automated layout planning. The research field of factory planning differs from related research topics such as facility layout planning (FLP) from operations research (OR) by focusing for example on qualitative criteria in the evaluation. However, findings on how qualitative criteria can be interpreted in a quantifiable and measurable way are still rare. In this article, we address these questions with a focus on changeability, which can be regarded as the most important qualitative evaluation criterion in layout planning. For this purpose, a survey was conducted that aims to determine changeability from the understanding of industry experts and to define it consistently with the understanding from literature. Based on this understanding, performance metrics are derived to measure the layout changeability.
\end{abstract}

Keywords Changeability quantification · Factory layout evaluation · Digital factory twin · Automated layout planning · Expert survey

\section{Introduction}

The growing market complexity, caused by shorter product and technology life cycles and customer individualized demands, is leading to a more intensive integration of new products into existing production processes [1]. Therefore, this also leads to an increase in the number of factory planning projects. The success of a factory planning project depends largely on the layout planning [2]. Layout planning is a complex planning problem that is currently characterized by many manual assessments and decisions [3]. Due to the volatile market environment of the factory, the flexibility and changeability can be regarded as one of the most important objectives in layout planning [4]. The changeable layout design allows an early anticipation of change requests and enables a quick and easy realization. By specifically addressing changeability as an objective, significant costs can be saved in case of a change, which quickly overcompensate for the additional costs initially incurred [5].

Viviane Hahn

v.hahn@wzl.rwth-aachen.de

$1 \quad$ Laboratory of Machine Tools and Production Engineering (WZL), RWTH Aachen University, Aachen, Germany
Many companies support their factory planning process with digital tools: digital design and simulation tools are already prevalently used in factory planning projects [2]. The available software provides more and more possibilities. For example, a digital factory twin, like the Integrated Factory Modelling (IFM) model, can be used in the planning process. This offers the advantage of being able to access more detailed information than a pure 3D visualization by also using information that can be assigned to objects [6].

The evaluation and choice of a layout alternative is usually based on a qualitative utility value analysis, which is developed in workshops or discussions. This requires a lot of time and assumes that the planning team has a high level of planning skills [7]. Due to the lack of an objective evaluation approach, there is also the risk that layout variants are selected which do not represent an optimal alternative with regard to the overall objectives [8].

Currently, evaluation processes are usually user driven. Many decisions are made based on human intuition and experience. This human intuition cannot be easily transferred into mathematical algorithms. On the way to automated layout planning, human behaviour must be understood and made machine interpretable first. For this purpose, twodimensional layout planning based on geometric data is no longer sufficient. For the inclusion of qualitative assessment 
variables, data, providing additional information to the geometrics of objects, must be included.

In doing so, the process of reverse engineering from the product development is adopted. Reverse engineering starts with an already existing product, which is disassembled first to extract construction parameters and elements and to achieve improvements [9]. Applied to the research field of layout planning, this means, that the evaluation parameters should be derived from the evaluation itself. Therefore, we conducted an exploratory survey to gain an improved understanding of human evaluation processes and make them measurable. The objective of the survey was to develop performance metrics for evaluating the layout changeability in an automated layout planning process. As an environment for the automated layout evaluation, the digital factory twin is used.

\section{Theory background}

\subsection{Definition flexibility and changeability}

According to WIENDAHL, flexibility refers to the predefined room for change, which enables adjustments without substantial changes to the factory structure. The factory's ability to change is limited by flexibility corridors defined at the time of planning. Within such flexibility corridors, fluctuations, e.g.in the number of produced units, can be compensated by means of measures provided (Fig. 1).[2]

Based on the definition of Wiendahl, two different concepts of flexibility can be differentiated in accordance with Browne-flexibility of volume and product flexibility. Volume flexibility indicates the extent to which volume related fluctuations in demand can be absorbed without causing an economic deficit. The product flexibility shows the extent to which the composition of the production program can vary without this having a negative impact on the system optimized production profit. In connection with the types of

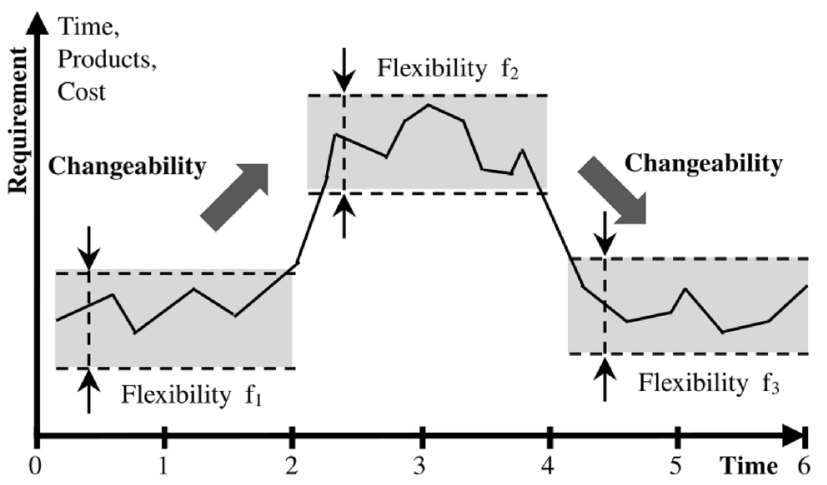

Fig. 1 Flexibility and changeability flexibility, BROWNE also mentions the expansion flexibility [10]. In the context of this article, expansion flexibility is represented by the concept of changeability.

According to WIENDAHL, changeability is the ability of a factory to actively change its structure with little effort, based on internal or external triggers [2]. The changeability allows for quick and cost-effective adaptations of the factory structure even beyond the predefined flexibility corridors. The design of a changeable factory is based on several changeability enablers, that support the factory's ability to change [2]:

- Scalability is characterized by the ability to expand and reduce functions regarding the available technology, space, and personnel.

- Modularity of a factory is characterized using standardized units that can function independently.

- Mobility is primarily understood as the ability to position objects within the factory. A high degree of mobility can be ensured by mobile machines and workplaces that can be rearranged without great effort.

- Compatibility is characterized by a high homogeneity of the media supply, and a particularly good networking capability of material and information flow.

- Universality describes the suitability of the factory to meet different requirements. This includes the spatial structure of the entire factory as well as individual operating resources.

\subsection{Layout evaluation of changeability}

In the field of factory layout planning, there are various approaches to layout evaluation. Due to many different target criteria, layout evaluation is a multi-objective evaluation problem with both quantitative and qualitative criteria such as the changeability of a factory.[11]

The utility value analysis, which is the most common approach used in factory planning practice, evaluates qualitative factors by means of a heuristic approach by transforming qualitative expressions of the target criteria into quantitative evaluation variables. The evaluation is based on semiquantifiable assessments by experts [12]. With the objective of evaluating different solution alternatives to a target system, it offers the possibility to create an evaluation in the form of a ranking based on the utility values [13]. The weighting is based on the specific factory target profile. As the quantification of qualitative variables is not an explicit approach and depends strongly on the skills and subjective viewpoint of the planner, it makes sense to carry out both the implementation and the interpretation of the utility analysis in a larger planning team with several specialist disciplines. Similarly, the achieved utility value 
of an alternative should not be seen as an absolute value, but rather in its relation to other results.[12]

Wesebaum et al. developed a method for quantitative layout evaluation considering qualitative objectives as well. The target criteria are evaluated by modelling the layout using elementary cells. An elementary cell is a discrete area element, which is uniquely defined by its coordinates and an identification number (ID). Besides the coordinates and the ID, elementary cells have other relevant attributes for factory planning. These can be, for example, load capacities, noise power levels or illuminance. Based on this information, the authors formulate specific calculation rules for estimating a layout variant regarding various target criteria. As target criteria for changeability Wesebaum et al. use the changeability enablers discussed above.[3]

In order to achieve an objective and quantitative approach to evaluate changeability of production systems, Schuh et al. use ERP feedback data. They use the dependency of change, time and effort to determine the degree of changeability. For this, feedback data has to be related to the components. The methodology has to be adapted to every production system as well as to the user preferences and the user specific definition of changeability. With the help of a tool, an automated calculation of predefined change-effort-ratios is enabled. The quantification of changeability can be done with the change enablers to determine the different ratios. For example, the ratio for "mobility" can be measured based on time and effort it takes change information and material flows. Furthermore, universality can be measured based on the variation of throughput time and the number of production processes.[14]

Klempke and Nyhuis have developed an approach to evaluate and design factories regarding lean and transformable factories. For this, requirements are defined at the basis of the changeability enablers. The requirements are derived from the factory levels (site, factory, cell, workstation) and fields (means, organization and space) for all factory objects. On a workstation level exemplary transformability requirements are given. In case of means, universally applicable production technologies are required. As a result, different products can be produced with the same technology. Additionally, the means should be mobile and easy understandable. For the field of space, adaptable lighting and interior climate conditions are required. Furthermore, workplaces should be adaptable with regard to ergonomic needs of the employees. They point out that a high level of standardization improves transformability. As a result, means of storage can be used at all workplaces without modifications. Based on the requirements, the factory objects are evaluated qualitatively and quantitatively. All values are transformed into a percentage scale to make the evaluated objects comparable. [15]
ABDI developed an approach for layout evaluation based on the analytical hierarchy process (AHP). The idea of the AHP methodology is to reduce decision complexity by considering individual hierarchical levels. It is used to map the complexity in layout planning resulting from a variety of objectives. The overall objective of the approach is to select the most changeable layout variant. Accordingly, this objective is placed on the highest hierarchical level. On the second level the influence of the decision maker on the selection of the layout variant is considered. The decision depends largely on whether the layout is selected by top management, the plant manager or the production engineer. To select the best layout variant, the author focuses on the sub objectives reconfigurability, costs, quality and reliability on the third level. Relevant target criteria are derived on the fourth level based on these superordinate objectives. Reconfigurability in this context can be equated in parts with the term changeability. The changeability of a layout can be characterized according to ABDI by for example the convertibility, scalability, modularity, mobility and conversion speed. At the lowest level of the hierarchy are the different layout variants, which serve as a basis for the evaluation of all preceding hierarchy levels. Overall, the approach enables the decisionmaking process to be systematized, particularly regarding qualitative target criteria. However, the explicit consideration of quantitative variables is not part of the evaluation process, as the evaluation of the target criteria is only based on pairwise comparisons.[16]

Summarising, different approaches to quantifying flexibility and adaptability for factory and layout structures already exist. However, the evaluation of changeability is often based on a manual, usually linguistic assessment by the planner and is therefore not applicable in the context of automated layout evaluation. Apart from the approach of Wesebaum et al., there are no approaches that are explicitly dedicated to the quantification of flexibility and changeability. Based on the insights gained, we will integrate the quantification-based assessment into the digital factory twin model and will ensure that the findings are implemented by experts. In order to achieve a practice-oriented quantification of the changeability and to make the complex evaluation processes comprehensible and measurable, the implementation of an expert survey was identified as a promising approach. This survey is presented in the next section.

\section{Methodology}

The objective of our survey was to understand manual evaluation processes and to quantify these based on geometric measurements. Based on the process of reverse engineering and the approach of mental simulation, the intention was to draw on the experience of experts and 
to understand their cognitive processes in the situation of decision-making and finally to reproduce them using geometric measurements [17]. The measurements were used to develop performance metrics for changeability. These metrics allows the evaluation to be performed completely digitally and automatically in a digital factory twin.

As a basis for modelling the performance metrics, guideline based expert interviews were conducted. Regarding to Bogner et al. guideline interviews pursue the objective of generating specific information on a research topic that could not otherwise be obtained.[18]

In our explorative expert survey following Bogner and Meuser, we had to ensure that the industrial understanding of changeability was defined consistently with the understanding from the literature, while leaving room for discussion of new aspects and ideas. We identified the method of semi-structured interviews as suitable for our purposes because it combines these requirements.[19]

\subsection{Presentation of survey design}

The objective of the interviews was to determine empirically which factors are included in the manual assessment of changeability to develop performance metrics based on these factors. For this purpose, seven experienced factory planners from research and industry were interviewed. The interview was conducted either by telephone or face-toface. A semi-structured interview guide was used as the basis for the survey. The division of the discussion with the interview guide was not strict but served as a rough guideline. Thus, the focus of the discussion could be set individually depending on the field of expertise of the participants. The questions were clustered into thematic blocks so that the respondent was not forced to make frequent mental leaps and could understand the logic of the interview process [20]. The interviews were divided into five parts:

\subsubsection{Introduction}

The first part of the interview did not contain any complicated questions, but rather questions that could be answered by the expert without much effort. These included background questions, such as demographic data, questions about the professional background and experience in the field of factory planning. In addition, open questions regarding the further process of the interview were answered. The interview guide, visualizations of layouts that the experts could refer to in the interview and a glossary to clarify terms were sent out in advance.

\subsubsection{Definition of flexibility and changeability}

The second part of the interview dealt with the industrial understanding of the two terms flexibility and changeability. The participants were asked to define the two terms. The aim was to capture the industrial understanding of flexibility and changeability and to compare it with the understanding from literature. In this way it should be ensured that the metrics correspond to the understanding of the potential user and are equally consistent with the understanding from the scientific literature.

\subsubsection{Planning levels of changeability}

The third part of the interview dealt with the issue of changeability at different planning levels of layout planning. In this case they are defined as the level of detail in which planning takes place. A distinction is made between the levels of plant structure planning, rough planning and detailed planning. After the participants had defined the terms flexibility and changeability, they were asked to assign the potential for change to the different planning levels of layout planning. The planning levels identified in this way later serve as a basis for identifying factors influencing changeability.

\subsubsection{Identification of evaluation parameters}

The fourth part of the interview dealt with how the experts currently assess layouts in terms of changeability and which parameters are used to measure it. As a basis for the quantification the experts were presented the changeability enablers: scalability, modularity, mobility, compatibility and universality. Subsequently, we asked whether these target criteria could also be measured geometrically in the layout. By capturing their understanding of flexibility and changeability, existing approaches could be validated and evolved in the required way. The objective was to link the evaluation directly to the geometry of the layout and thus enable automation.

\subsubsection{Open questions}

The fifth part of the interview dealt with the open questions left. The participants had the opportunity to comment on the topics covered in the interview and to express ideas and criticism. In addition, other potential experts for the survey were identified.

The introduction lasted about 10 min, depending on the number of questions from the participants. For all participants, the interviews lasted about one hour each. We recorded the discussions with two separate voice recorders. The interviews were analysed using the software MAXQDA 2020. It enables the coding of data through qualitative 
content analysis. The analysis was carried out according to the "grounded theory" using the approach developed by Meuser and Nager. Hence, the stages transcription, coding and thematic comparison with subsequent conceptualization and generalization in the form of abstracted metrics were followed [21]. The objective was to analyse the transcripts of expert interviews in a purposeful way to derive conclusions for answering the research questions. For this purpose, we categorized the findings with the following tags: "definition flexibility", "definition adaptability", "scalability", "modularity", "mobility", "compatibility" and "universality" on the different planning levels of layout planning.

\subsection{Presentation of the survey sample}

In this section, the survey sample is discussed. A total of seven experts participated in the survey. The participants were recruited from both research and industry. They were included based on their experience in factory and layout design to ensure that they have the same expertise and professional background. Most of the contacts were established by the working group "Factory Planning" of the Laboratory for Machine Tools and Production Engineering (WZL) of RWTH Aachen University.

\section{Key findings}

This section summarizes the major findings of the expert survey. The findings result either directly from the feedback of the survey participants or indirectly from requirements that were identified through the discussions. First, our findings regarding the industrial understanding of flexibility and changeability is explained and, based on that, future challenges on the way to automated layout planning are derived.

\subsection{Definition of flexibility and changeability}

The results of the survey show that it is very difficult to differentiate precisely between the objectives of flexibility and changeability. About $70 \%$ of the experts interviewed are struggling with the mentioned differentiation. This is because both flexibility and changeability are often understood as the adaptability of the factory structure. Thus, the understanding in practise essentially coincides with the understanding from scientific literature, which is shown in Fig. 2.

In contrast to changeability, the experts localize flexibility within the given factory structure. The adaptability of the factory structure is characterized by most experts through their relation to the product or the equipment used. Flexible factory structures must be able to handle a certain amount

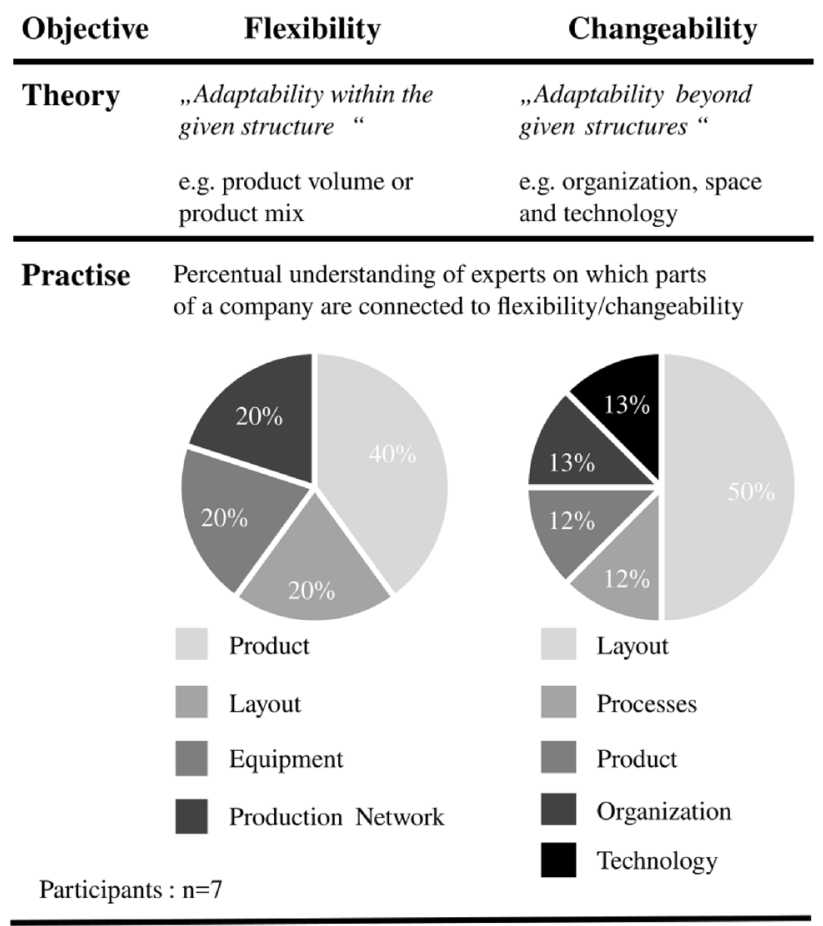

Fig. 2 Practical and theoretical understanding of flexibility and changeability

of variance in the product range, for example in volumes or in the product mix. This is usually achieved through the equipment used, e.g. how easy it is to exchange or replace aggregates of a facility.

The experts identify the increasing market dynamics as a key driver of change. More and more frequently, the need arises to be able to make adaptations to the factory beyond the given structure. Shorter product life cycles lead to an increasing integration of new products into the production program. This leads to the need to extend existing production lines or plan new lines, i.e. a substantial change of the factory structure.

The adaptability beyond the given structures is characterized both in theory and by most of the experts about the factory's changeability. Despite foresighted planning and the analysis of different scenarios, it is a big challenge for planners to estimate such transformation needs in advance. This makes it even more important to ensure the changeability regarding unplanned change requirements already at an early factory planning stage, e.g. by keeping expansion or reduction areas available.

More than half of the experts interviewed localize the changeability in the factory rough layout. A change is therefore usually expressed by shifting departments. This requires that different functions can grow into the existing space of the factory without requiring complex or costly adjustments. Influencing factors can be, for example, the building 
foundations or the media supply, which must be designed in advance with a perspective of a future change scenario.

The explanations show that flexibility can be distinguished from changeability both in theory and in practice and has a different value for layout planning. Flexibility is essentially based on adaptability within the given factory structure. Since flexibility is primarily represented by the flexibility of the equipment, it can be regarded as a step prior to layout planning. Therefore, metrics for quantifying changeability were developed further.

The identification of evaluation parameters was the most difficult task for the experts. Especially the identification of the influence of underlying geometric parameters to the factory layout. For the experts it was easier to identify connected data that have to be considered in layout evaluation. The resulting information were consolidated to indictors for the layout evaluation and used for the development of the metrics described in the following paragraphs.

\subsection{Challenges on the way to automated layout planning}

In general, the interviewed experts attested the automated layout planning a high potential. Quantifying layout planning objectives is one of the major challenges on the way to automated layout planning. In this context the conducted survey first analyses the flexibility and changeability. The expert understanding of these objectives serves as a basis for the derivation of target criteria of the underlying objective system and indicators for the development of metrics for the evaluation of these target criteria. The explicit development of the objective system and metrics were not part of the survey and were developed based on the results.

\subsubsection{Development of an objective system}

One challenge of automated layout planning is to develop an objective system. If the precision of the objectives and target criteria is too low, there is a risk that the evaluation approach will be influenced by certain simplifying assumptions. Such simplifications become particularly problematic if they do not correspond to the user's understanding of the underlying objectives.

The expert survey shows that the understanding of changeability can significantly differ between different experts from the industry. Therefore, it is important to formulate the target criteria of the objective system as clearly as possible. If the resolution of the objective system is too low, it cannot be guaranteed that the evaluation provided represents the evaluation in the sense of the user.

There is a need to clarify the objective of changeability and to subdivide it once again into different target criteria. These target criteria can be prioritised individually and project-specifically to be able to map an individual understanding of changeability. To derive the target criteria of changeability a systematic approach is required. The approach of Glose et al. discussed earlier, uses the AHP for structuring target criteria. Such a hierarchical structuring should also be the basis for the development of performance metrics in this article. The horizontal decomposition of changeability is carried out by dividing it into the target criteria scalability, modularity, mobility, compatibility and universality, which also are considered as change enablers in literature. The target criteria are shown in Fig. 3.

Based on this structure the survey participants identified measurement parameters and corresponding performance metrics, which will be presented in detail below. On the way to automated layout planning, numerous other objectives and target criteria must be quantified and modelled using appropriate target criteria in addition to the changeability. The investigation of the changeability was a first step on this way and should show that the quantification of qualitative objectives is basically practicable.

\subsubsection{Develop performance metrics}

The next section discusses the performance metrics for the changeability, which were developed based on the input of the survey participants and the findings of the literature review. In connection with the formulation of metrics, two major conclusions can be outlined:

1. The development of metrics on a geometrical level is only possible to a limited extent due to the planning complexity of layout planning.

2. On a parametric basis, the integration of layout-relevant data results in extended possibilities for evaluation.

Although the experts localized changeability at all planning levels of layout planning as an objective, the most significant benefit was first identified at the rough layout level.

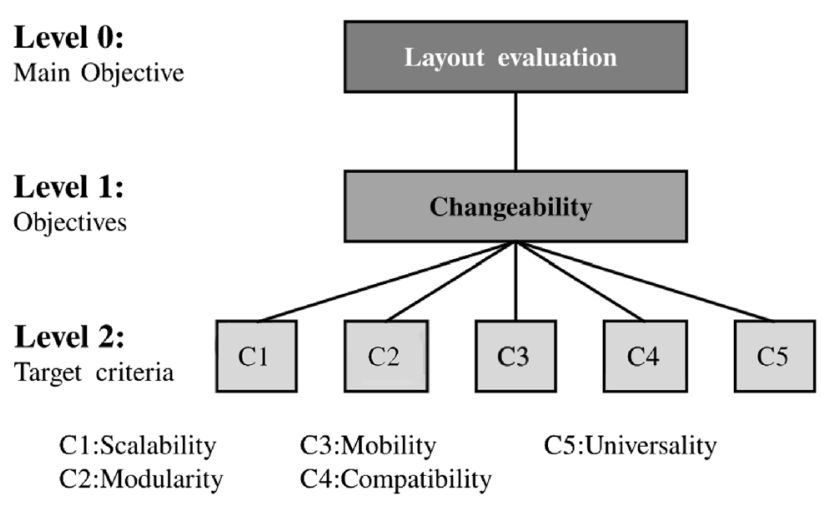

Fig. 3 Objective system of changeability 
In addition, by considering the rough layout level, the planning complexity and the degrees of freedom can be reduced to such an extent that they can be better represented in the context of a digital evaluation process.

In addition to the layout geometry, further layout-relevant data is required to evaluate changeability. The additional data makes it possible to interpret the geometry of the layout in terms of the performance metrics and thus provide an assessment regarding the proposed target criteria. The aim of the assessment is to make different layout variants comparable in terms of the target criteria. For this reason,

\section{Layout sample scalability}
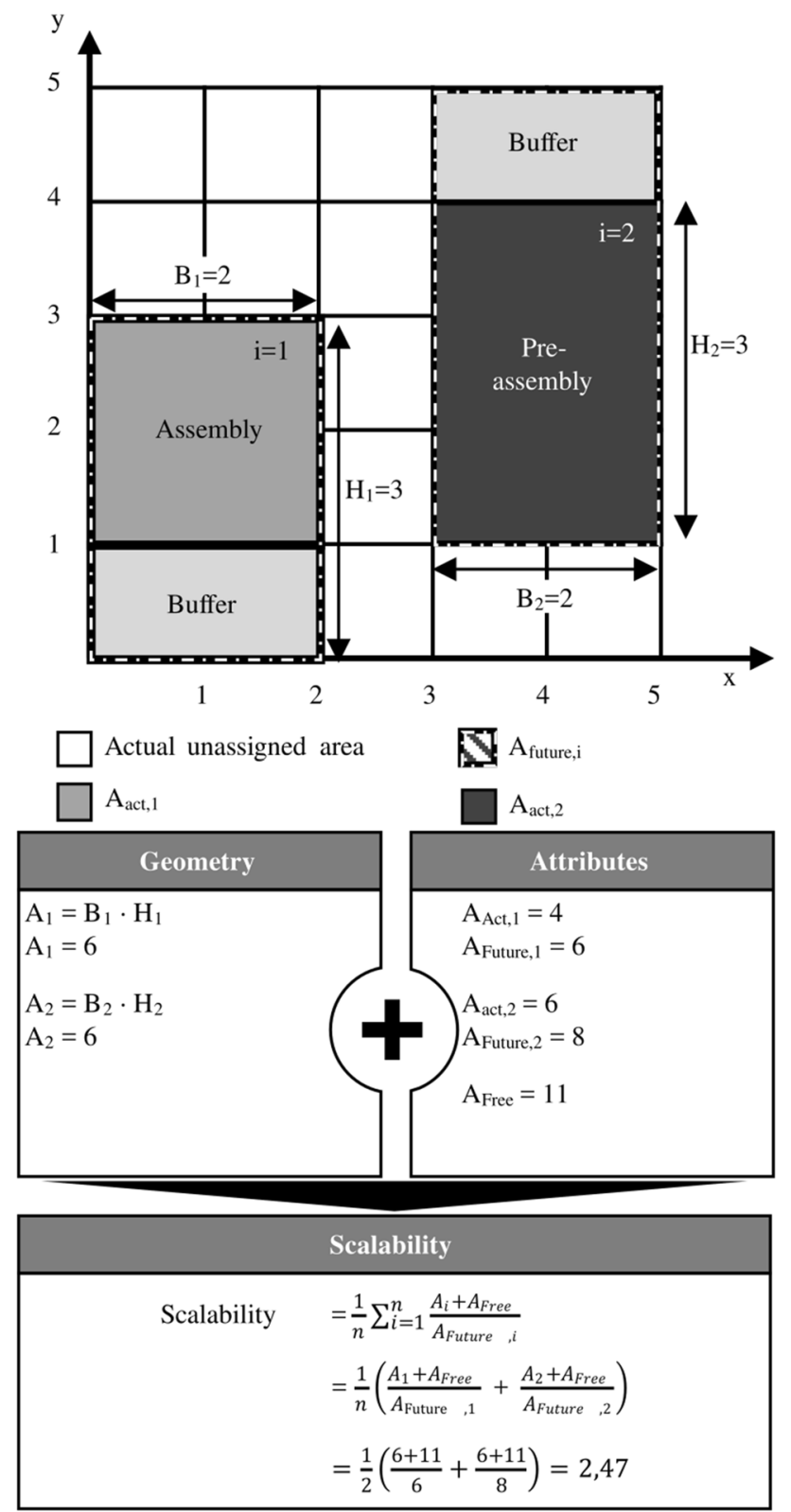

Fig. 4 Evaluation of scalability the absolute values of the respective evaluation result are not the decisive ranking criterion for the variants, but the relative classification of the layouts to each other based on their values.

\subsubsection{Scalability (C1)}

In the interviews, most of the statements regarding the layout scalability referred to existing free or reduction areas. Keeping space available makes it possible to build up new technologies or to expand or reduce existing technologies in a meaningful way. In addition to the availability of space, the expandability or reduction of the media grid plays a decisive role in this context. The scalability of a layout can be calculated with formula 1.

Scalability $\mathrm{C} 1=\left\{\begin{array}{ll}\frac{1}{n} * \sum_{\mathrm{i}=1}^{\mathrm{n}} \frac{A_{\mathrm{i}}}{A_{\text {Future, }},} & \text { if } \frac{A_{\text {act, } \mathrm{i}}}{A_{\text {Future, }}}<1 \\ \frac{1}{n} * \sum_{\mathrm{i}=1}^{\mathrm{n}} \frac{A_{\text {Future, } \mathrm{i}},}{A_{\mathrm{i}}}, & \text { if } \frac{A_{\text {act, } \mathrm{i}}}{A_{\text {Future, }}}>1\end{array}\right.$,

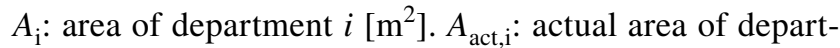
ment $i\left[\mathrm{~m}^{2}\right] . A_{\text {Future, },}$ : future space requirement of department $i$ calculated in growth scenarios $\left[\mathrm{m}^{2}\right] . A_{\text {Free, } i}:$ Free area which is not assigned in growth scenarios $\left[\mathrm{m}^{2}\right] . n$ : number of departments [-].

To evaluate scalability, the area of a department is set in relation to the predicted future space requirements. According to an expert, the forecast of future space requirements can be based on possible production scenarios. The space requirement depends on the product mix and production quantity, among other things. Buffer or reduction areas can be defined, depending on whether growth or a decrease in space requirements is assumed. If all estimated future space requirements of possible growth scenarios have already been realized at the time of planning, the layout can be considered as planned scalable. If there are additional free areas beyond the future growth scenario areas, the layout will be evaluated as scalable for unplanned scenarios as well. The higher the value of this metric is, the more scalable is the layout. The exemplary application of the metric is illustrated in Fig. 4.

\subsubsection{Modularity (C2)}

Most of the experts identified modularity as one of the central drivers of changeability. If there is a need for change, the modular structure allows existing modules to be expanded or exchanged. In this way, the factory structure can be changed easily and cost-effectively. The layout modularity can be calculated as follows:

Modularity $C 2=\frac{\sum_{m=1}^{n} A_{m}}{A_{\text {Overall }}}$, 

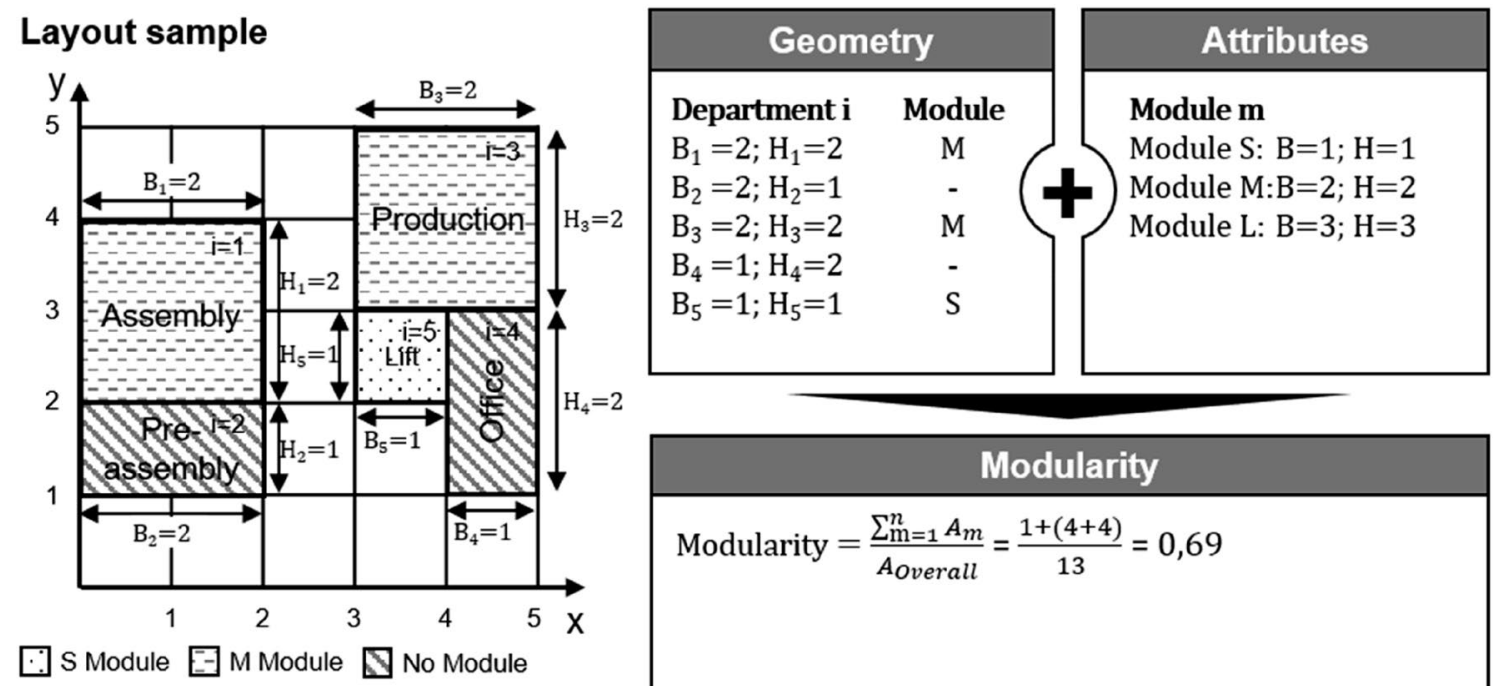

\begin{tabular}{|c|}
\hline Modularity \\
\hline Modularity $=\frac{\sum_{\mathrm{m}=1}^{n} A_{m}}{A_{\text {Overall }}}=\frac{1+(4+4)}{13}=0,69$ \\
\hline
\end{tabular}

Fig. 5 Evaluation of modularity

$A_{\mathrm{m}}$ : area with module type $\mathrm{m}\left[\mathrm{m}^{2}\right] . A_{\text {Overall }}$ total used area $\left[\mathrm{m}^{2}\right] . n$ : number of module types [-].

One expert remarked that there is the possibility to define modules spatially. For this purpose, a limited number of modules can be defined beforehand. These modules can then be easily exchanged when a change is required. Other authors define modules as independently functional units. However, the consideration of independently functioning modules is an additional planning restriction because the authors require the modules to have the same structure [22]. This restriction can be avoided by defining modules spatially. The evaluation of the modularity, however, is based on the ratio of the modularly planned and the total area of all areas. With a value of one, the entire area is modularly planned. The exemplary application of the metric is illustrated in Fig. 5. The higher the value of this metric is, the more modular is the layout.

\subsubsection{Mobility (C3)}

One of the experts stated that mobility is regarded as a fundamental prerequisite for modularity, since only through the mobility of modules existing structures can be changed. Consequently, it seems reasonable to also think in terms of mobility in modules. Departments with a high degree of mobility should be spatially concentrated, since within this

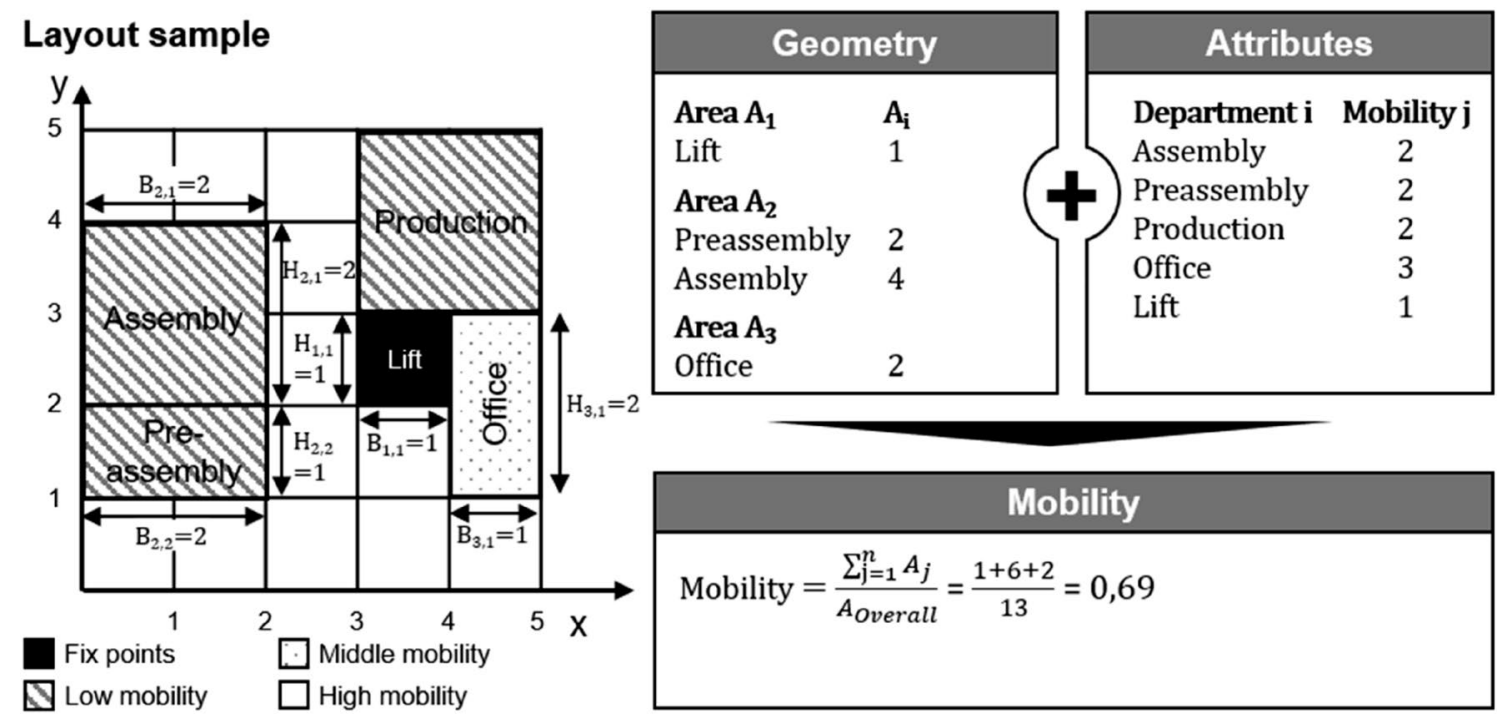

Fig. 6 Evaluation of mobility 
area adjustments can be made with little effort, e.g. to react to a change in the product mix. Areas with low mobility should be spatially aggregated so that they do not hinder more mobile areas in the event of potential change [23]. The mobility potential of a layout can therefore be evaluated by the size of the connected areas with the same mobility. The mobility potential can be calculated using formula 3 :

Mobility $C 3 a=\frac{\sum_{j=1}^{n} A_{j}}{A_{\text {Overall }}}$,

$A_{\mathrm{j}}$ : maximum coherent area with mobility degree $j\left[\mathrm{~m}^{2}\right]$. $A_{\text {Overall: }}$ total used area $\left[\mathrm{m}^{2}\right] . n$ : number of module types [-].

\section{Layout sample fix points}
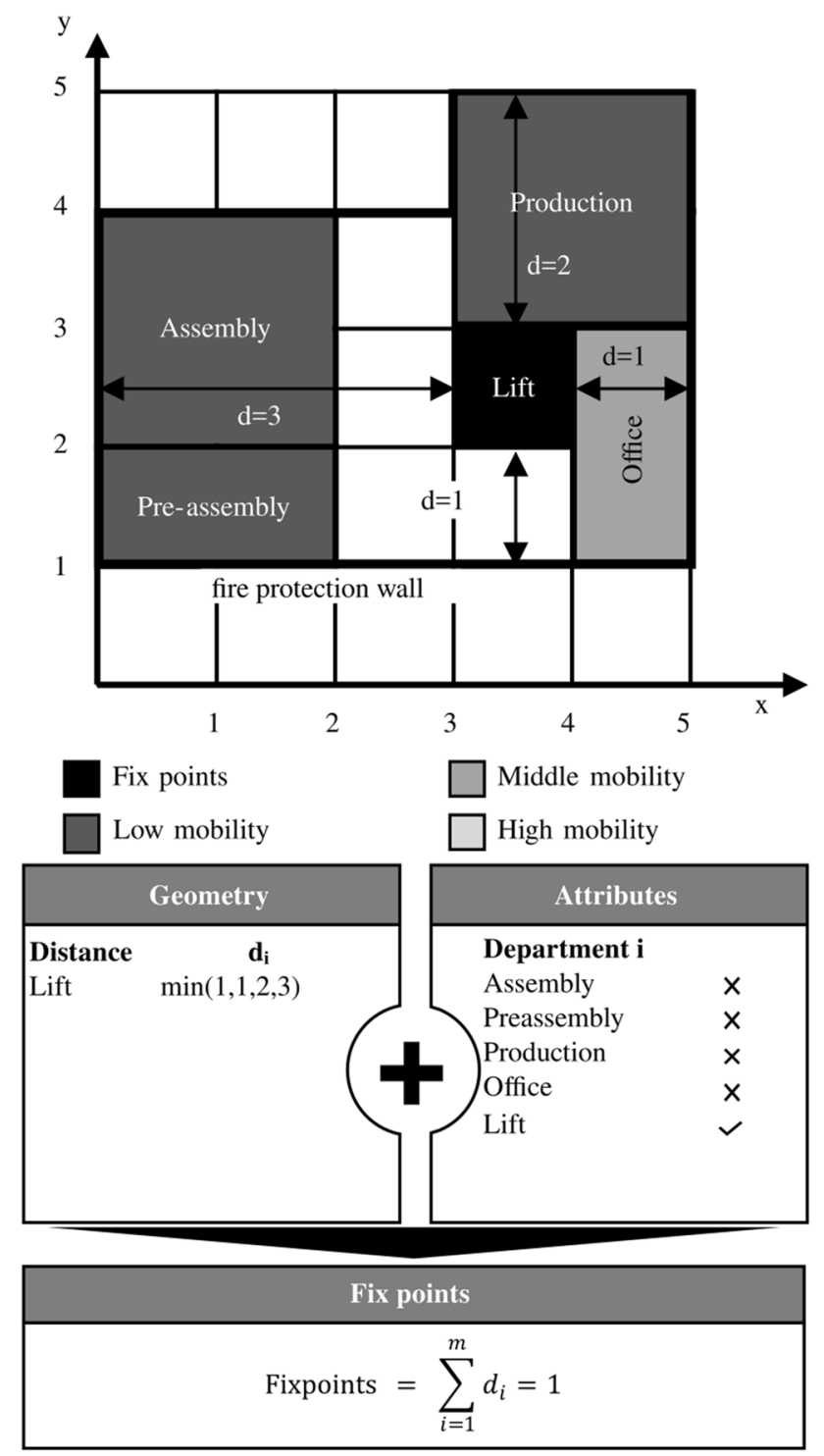

Fig. 7 Evaluation of fix points
Each department is considered as a module with a certain mobility level j. Several experts stated that the mobility of an area is determined mainly by the equipment available. In this context, one expert suggested, that workstations can be positioned on rolls and thus rearranged easily when the product mix or volume changes. This makes it possible for light equipment to achieve a high degree of mobility and to react flexibly to any changes. The exemplary application of the metric is illustrated in Fig. 6. The higher the value of this metric is, the more mobile is the layout.

In addition to the spatial aggregation of areas with the same mobility, it also makes sense to consider immobile areas, so-called fix points, separately. Monuments are a restriction in case of change. Since restrictions considerably restrict the solution space, their influence on the mobility of a layout must be minimized. The influence of the monuments can be calculated with formula 4 :

Fix points $C 3 b=\sum_{i=1}^{m} d_{i}$,

$d_{\mathrm{i}}$ : shortest distance to a static or fire protection wall [m]. $m$ : number of fix points [-].

According to an expert, the influence of a monument can be minimized by placing it next to static or special walls, like fire protection walls, of the layout. The shortest distance of a fix point to the nearest static wall can be used as a measurement. The distance to the nearest static or fire protection wall is considered, because lightweight construction walls can be cost-effectively removed with low effort. This essentially corresponds to the understanding developed by HEGER regarding the advantageous positioning of fix points at the edge of the layout [24]. The position of the factories wall is defined by the coordinates of the assigned departments. For this purpose, the maximum and minimum values of all departments in X-and Y-direction are analysed. Figure 7 illustrates the exemplary application of the metric for evaluating fix points. The lower the value of this metric is, the more mobile is the layout.

\subsubsection{Compatibility (C4)}

The layout compatibility was defined within the framework of the conducted expert survey on the most homogeneous possible media availability. The analysis of the interviews shows that the size of the media grid can be used as a measurement in the evaluation of a layout alternative. The mesh size of the media grid can be evaluated both spatially and functionally. Spatially in this context means how far apart the connections of the media grid are. One of the experts stated that the closer the mesh size, the higher the adaptability of a layout can be considered. In the ideal situation, complete media availability is given at every location of the 
factory. Other authors evaluate the compatibility by deviating from this ideal state.[23]

However, the interviewed experts noted that this can hardly be realized in practice for cost reasons. For this reason, media required for a conversion are usually installed subsequently. For this purpose, the corresponding prerequisites must be met in advance. Several experts explained the necessity of standardized interfaces in the media grid. These enable a quick coupling of areas with different media availability or simple retrofitting. Furthermore, it seems to make sense to spatially aggregate areas with the same media requirements to achieve a homogeneous distribution of the media. The compatibility can be calculated with formula 5:

Compatibility $C 4=\frac{\sum_{p=1}^{n} A_{p}}{A_{\text {Overall }}}$

$A_{\mathrm{p}}$ : maximum coherent area with media profile $p\left[\mathrm{~m}^{2}\right]$. $A_{\text {Overall }}$ : total used area $\left[\mathrm{m}^{2}\right] . n$ : number of media profiles [-].

Areas with the same requirements for media supply must therefore be spatially concentrated. This makes it possible to locate equipment with specific media requirements quite unrestrictedly, since the available space is at a maximum. In order to make an estimation of the spatial aggregation of the areas, the sum of the largest area with the same media profile can be related to the total planned area. Figure 8 illustrates the exemplary application of the metric for compatibility. The higher the value of this metric is, the more compatible is the layout.

\subsubsection{Universality (C5)}

Most of the experts define the universality of a layout based on the possibility of reusing the existing factory structure. However, such a change of use is carried out rather radically in business practice. The planning of universal buildings is usually not done in practice, or only to a very limited extent, for cost reasons.

Furthermore, the expert survey shows that the target criteria universality and compatibility are difficult to differentiate. This is since the conversion of areas also requires a connection to the media grid. In the context of this article, the differentiation proposed by Wesebaum et al. will be used as a guide. The authors do not relate the universality to media supply, but to the basic building structure. The universality of the building structure can be assessed based on several structural factors, such as the building shape, homogeneity of the ceiling load-bearing capacity and the height profile. For further explanations on the quantification of these factors, please refer to the authors' work. [23]

In addition to the factors identified by Wesebaum et al., according to one of the experts interviewed, the homogeneity of the floor load-bearing capacity must also be considered in connection with universality. This makes it possible to relocate machines within these areas or to replace them if they expand from the area initially planned. Such an evaluation of the floor load capacity can be made using formula 6:

Load capacity $C 5=\frac{\sum_{t=1}^{n} A_{t}}{A_{\text {Overall }}}$,

$A_{\mathrm{t}}$ : maximum coherent area with load capacity $t\left[\mathrm{~m}^{2}\right]$. $A_{\text {Overall }}$ : total used area $\left[\mathrm{m}^{2}\right] . n$ : number of media profiles [-].

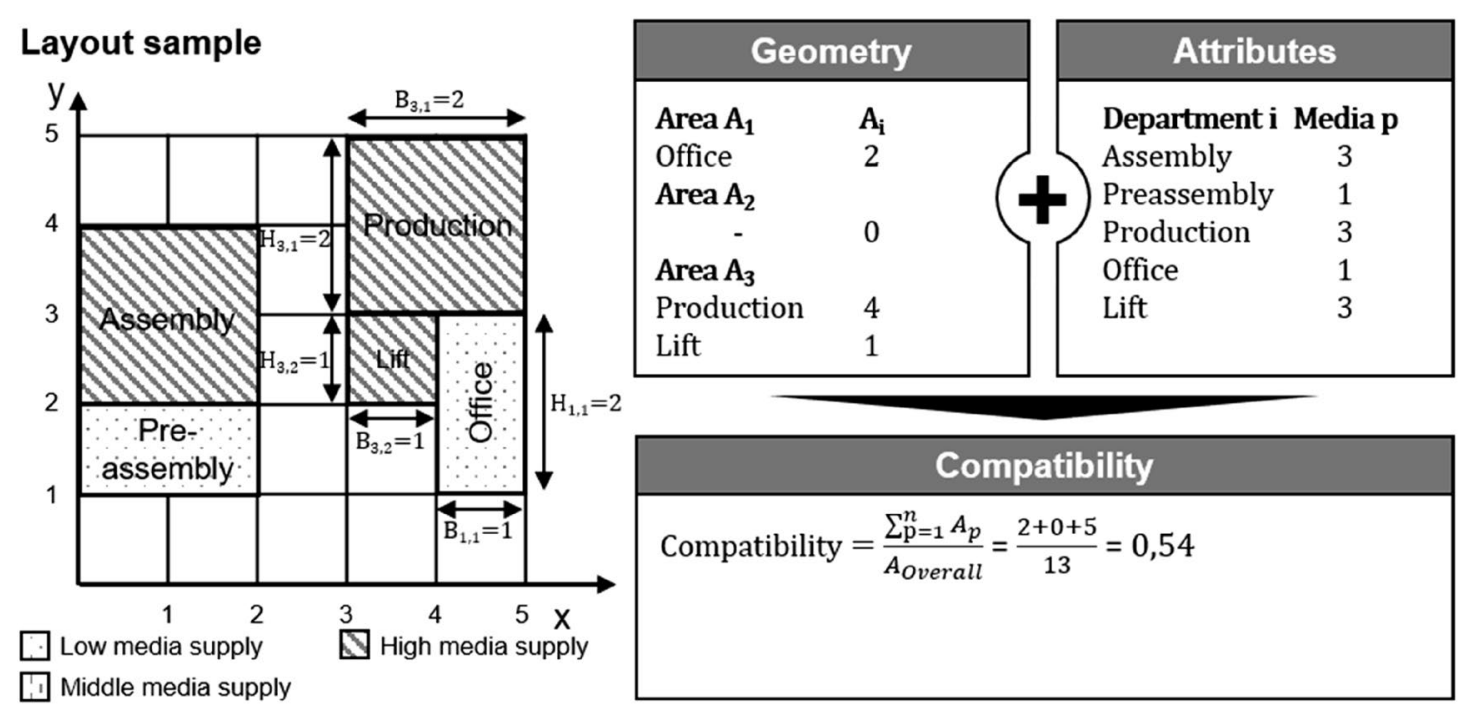

Fig. 8 Evaluation of compatibility 


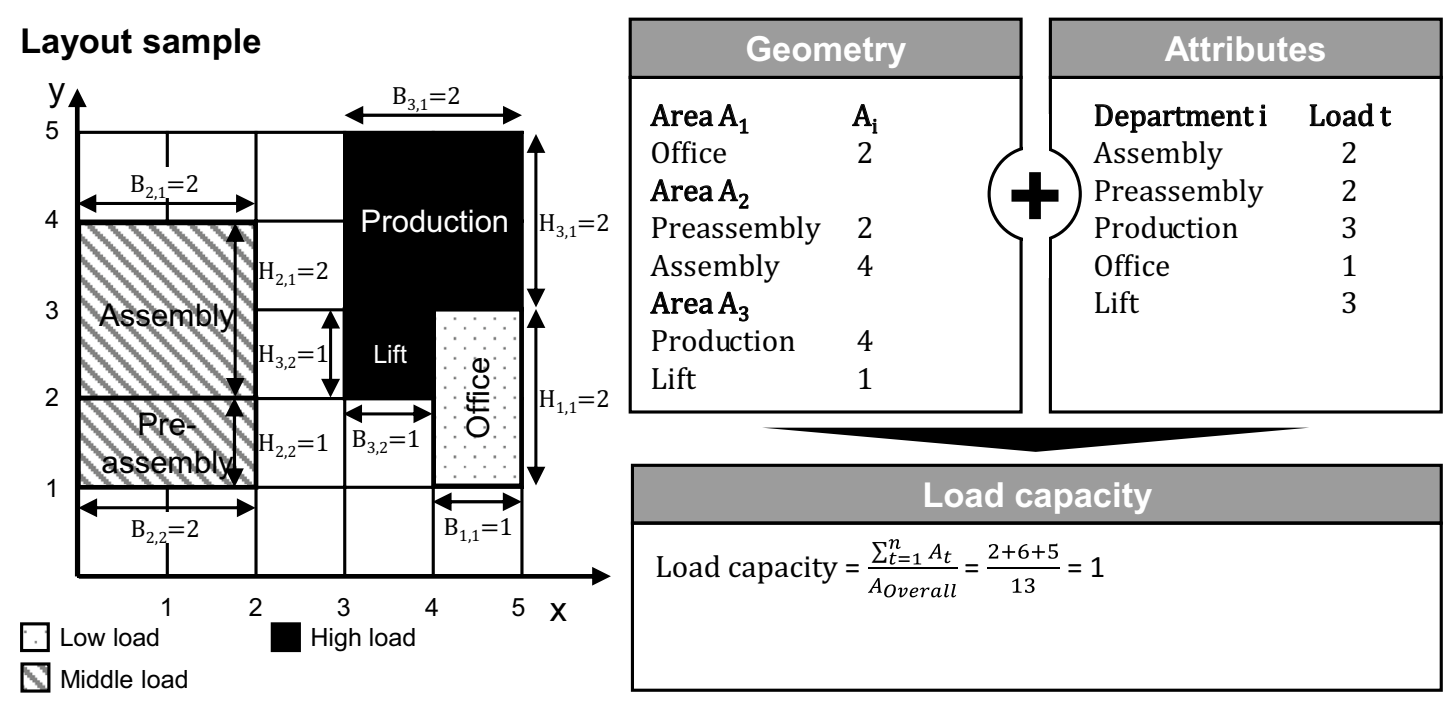

Fig. 9 Evaluation of load capacity

The homogeneity of the load capacity is expressed, analogous to the potential for compatibility, by spatially merging areas with the same load capacity needs. An estimation can be given by the ratio of aggregated and total area. Figure 9 shows the exemplary application of the load capacity metric. The higher the value of this metric is, the more universal is the layout.

\subsubsection{Derivation of the target function value}

In order to use the values derived from the presented metrics to transfer different layout variants into a ranking, a resulting target function value must be determined for each layout. It is not trivial to set up an objective function for the qualitative objective of changeability, which is made up of the presented target criteria in aggregated form. With the Technique for Order Preference by Similarity to Ideal Solution (TOPSIS) the objective function value for the entire changeability can be determined without knowing the explicit objective function. For this purpose, only the values of the individual target criteria functions, as determined by the application of the metrics, are required. The TOPSIS method [25] is divided into five steps. In the first step the normalized decision matrix is constructed. For this purpose, the previously presented metrics are used to determine the characteristics for the respective layout variant. Afterwards these are inserted into the decision matrix $\mathrm{E}$ (formula 7).

$$
\begin{aligned}
& \begin{array}{lllll}
C_{1} & C_{2} & C_{3} & C_{4} & C_{5}
\end{array} \\
& E_{1}=\begin{array}{c}
A_{1} \\
\ldots \\
\ldots \\
A_{n}
\end{array}\left[\begin{array}{ccccc}
c_{11} & c_{12} & c_{13} & c_{14} & c_{15} \\
\ldots & \ldots & \ldots & \ldots & \ldots \\
\ldots & \ldots & \ldots & \ldots & \ldots \\
c_{n 1} & c_{n 2} & c_{n 3} & c_{n 4} & c_{n 5}
\end{array}\right]
\end{aligned}
$$

$A_{\mathrm{i}}$ : layout variant $n . C_{j}$ : target criteria of changeability. $C_{\mathrm{ij}}$ : calculated value $i$ by use of the metrics for target criteria.

Afterwards the decision matrix has to be normalized. This is necessary because the target criteria can have different scales. While the evaluation of the fixed points is carried out, for example, via the underlying distance $[m]$, the mobility in a layout is estimated via the size of the areas $\left[\mathrm{m}^{2}\right]$ with the same degree of mobility. To normalize the decision matrix, vector normalization can be used in the TOPSIS method.[26]

$\tilde{c}_{i j}=\frac{c_{i j}}{\sqrt{\sum_{j=1}^{n} c_{i j}^{2}}}$,

$\tilde{c}_{i j}$ normalized target criteria value.

The second step is the weighting of the decision matrix. A specific approach for weighting is not given within the TOPSIS method. It is therefore considered appropriate to use the procedure of pairwise comparisons, which is common in factory planning. The weighting of the criteria is based on a series of pairwise comparisons. This procedure does not conflict with the goal of automating the evaluation. The different preferences could, for example, initially be specified 
by the planner in the overall vision of automated layout planning via a graphical user interface. This would eliminate the need for manual intervention in the process. The thus determined weightings of the goal criteria are transferred into a weighting vector $\vec{g}=(g 1, g 2, \ldots, g 5)$. For weighting, each column vector of the normalized decision matrix is multiplied by the weighting vector. $A_{\mathrm{i}}$ : layout variant $n . C j$ : target criteria $j$ of changeability. $\tilde{c}_{i j}$ normalized target criteria value. $\vec{g}$ : weighting vector.

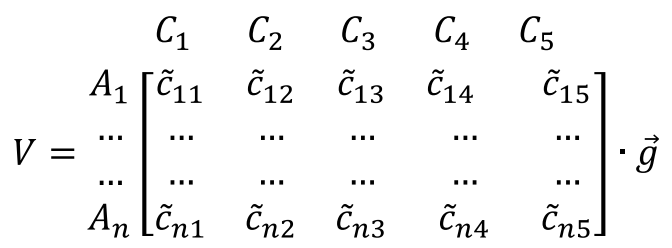

In step three, two virtual alternatives are built: a best-case solution combines the best criteria values of all layout variants, the worst-case solution the worst values. Furthermore, step four includes the calculation of the distance dimensions. For this purpose, the Euclidean distances of the real to the virtual alternatives are calculated. Euclidean distances measure the distance between two points in a multidimensional space. The dimension of the space is determined by the number of target criteria. The formulas for the calculation of the distance dimensions are presented below in formula 10 .

$$
\begin{aligned}
S_{i^{-}} & =\sqrt{\sum_{j=1}^{m}\left(v_{i j}-v_{j}^{-}\right)^{2}} \forall i=1, \ldots, n \\
S_{i^{+}} & =\sqrt{\sum_{j=1}^{m}\left(v_{i j}-v_{j}^{+}\right)^{2}} \forall i=1, \ldots, n,
\end{aligned}
$$

$S_{i^{-}}$: distance of layout variant $i$ to worst-case solution. $S_{i^{+}}$: distance of layout variant $i$ to best-case solution. $v_{i j}$ : value of target criteria $j$ for layout variant $i . v_{j}^{-}$: value of target criteria $j$ of worst-case solution. $v_{j}^{+}$: value of target criteria $j$ of worst-case solution.

In step five the distance index is determined. This index is based on the idea that an efficient layout variant should be as close as possible to the best case solution and as far away as possible from the worst case solution. The distance index $\mathrm{D} i *$ is consequently a measurement for the changeability of a solution. It can be determined by the following formula 11 .

$D_{i}^{*}=\frac{S_{i^{-}}}{S_{i^{+}}+S_{i^{-}}}$,

$D_{i}^{*}$ : distance index layout variant $i . S_{\left(i^{-}\right)}$: distance of layout variant $i$ to worst-case solution. $S_{i^{+}}$: distance of layout variant $i$ to best-case solution.

The distance index of changeability determined by TOPSIS corresponds to the respective target function value. A summarizing overview over the procedure based on ZHANG ET AL. is given in Fig. 10 [27].

The distance index takes a value between 0 and 1 . Good solutions will be close to the value 1 , bad solutions to the value 0 . The target function values determined in this way can be used for ranking the layout variants.

\section{Conclusion and outlook}

The increasing planning complexity, induced by shorter product and technology life cycles as well as an increasing customer individualization, require a higher frequency of new and redesigned factory layouts. Layout planning is a multi-objective planning problem in factory planning. Many companies must face the dilemma of having to cope with increasingly complex planning in ever shorter time.[28]

The automation of layout planning promises great potential. For the automation, however, the objectives and target criteria of layout planning must be quantified first. The analysis of existing approaches and the conducted survey showed that the layout changeability can be evaluated partially automated based on specific geometric measurement parameters and further attributes, which are linked to the departments being planned. The development of performance metrics forms the basis for automated layout planning. In combination with multicriteria decision methods, the metrics make it possible to directly link the evaluation of qualitative objectives to the layout geometry. For this purpose, multi-criteria evaluation approaches such as TOPSIS can use the calculated values of the metrics to evaluate the created layouts and hence identify the best layout.
Fig. 10 Determination of the target function value of layouts variants

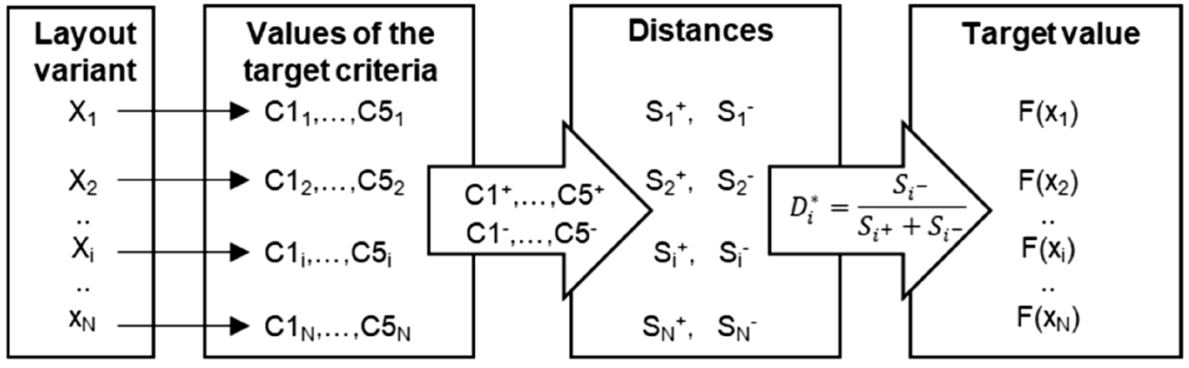


Nevertheless, the future work should include a representative survey with a representative number of participants from industry and scientific sector to validate the results and be able to make distinctions between company size and industries. Moreover, based on the results of the representative survey, a benchmarking survey should be performed to compare between the results of a human experts and the automated, metric based evaluation of changeability of the layout variants.

Further future work could contain to transfer the gained understanding to other qualitative and mapped in the form of suitable performance metrics. During the expert interviews, the objectives of security and organizational structure were discussed several times in this context. It is important that the planners are given the finest possible subdivision of the sub-criteria with clear definitions, as there is no consistent understanding of most qualitative criteria in layout planning. In this way it can be prevented that the evaluation results are distorted due to an unclear definition of the target criteria.

In connection with the qualitative objectives and target criteria of layout planning, there are also those that are highly subjective and therefore cannot be quantified, such as aesthetics. Since such criteria must also be considered in a holistic approach, it seems sensible to develop hybrid approaches, in which algorithms and human interact.

Consequently, concepts need to be developed for the collaboration of factory planner and algorithm. It is to be investigated how the use of interactive algorithms can relieve the factory planner. The evaluation of such subjective layout criteria can then be done manually by the factory planner. Based on the assessments made, the algorithm learns and can generate new, improved layouts.

Acknowledgements We would like to thank the experts who, with their expertise in the course of the survey conducted, have laid the foundation for the development of the results of this publication.

Funding Open Access funding enabled and organized by Projekt DEAL.

Open Access This article is licensed under a Creative Commons Attribution 4.0 International License, which permits use, sharing, adaptation, distribution and reproduction in any medium or format, as long as you give appropriate credit to the original author(s) and the source, provide a link to the Creative Commons licence, and indicate if changes were made. The images or other third party material in this article are included in the article's Creative Commons licence, unless indicated otherwise in a credit line to the material. If material is not included in the article's Creative Commons licence and your intended use is not permitted by statutory regulation or exceeds the permitted use, you will need to obtain permission directly from the copyright holder. To view a copy of this licence, visit http://creativecommons.org/licenses/by/4.0/.

\section{References}

1. Schuh G, Prote JP, Molitor M, Sauermann F, Schmitz S (2019) Databased learning of influencing factors in order specific transition times. Procedia Manuf 31:356-362. https://doi.org/10.1016/j. promfg.2019.03.056

2. Wiendahl H-P, Reichardt J, Nyhuis P (2015) Handbook factory planning and design, 25 edn. Springer Berlin Heidelberg, Berlin, Heidelberg

3. Wesebaum S, Mach F (2016) Fabrikbewertung durch mathematische Modellierung. ZWF Zeitschrift für wirtschaftlichen Fabrikbetrieb 111:100-103

4. Shariatzadeh N, Sivard G, Chen D (2012) Software evaluation criteria for rapid factory layout planning, design and simulation. Procedia CIRP 3:299-304. https://doi.org/10.1016/j.proci r.2012.07.052

5. Wiendahl H-P, Heger CL (2004) Justifying changeability. A methodical approach to achieving cost effectiveness. J Manuf Sci Prod 6:33-40

6. Burggräf PEA (2020) Integrated factory modelling-enabling dynamic changes for the factory of the future at the example of e.GO Mobile AG. In: Conference on Future Production of Hybrid Structures, Wolfsburg, Germany 27-28th May 2020 \%8, Wolfsburg, Germany

7. Tisch M, Laudemann H, Kreß A, Metternich J (2017) Utilitybased configuration of learning factories using a multidimensional, multiple-choice knapsack problem. Res Exp Educ 9:25-32. https://doi.org/10.1016/j.promfg.2017.04.017

8. Schuh G, Reuter C, Brambring F, Luckert M (2017) Predicting the potential of production structure changes using characteristic curves. Prod Eng Res Devel 11:125-131. https://doi.org/10.1007/ s11740-016-0709-0

9. Chikofsky EJ, Cross JH (1990) Reverse engineering and design recovery: a taxonomy. IEEE Softw 7:13-17. https://doi. org/10.1109/52.43044

10. Browne J, Dubois D, Rathmill K, Sethi SP, Stecke KE (1984) Types of flexibilities and classification of flexible manufacturing systems

11. Dannapfel M, Vierschilling SP, Losse S, Matzke O (2020) Generative design in factory layout planning: an application of evolutionary computing within the creation of production logistic concepts. In: 1st Conference on Production Systems and Logistics

12. Hermann M, Winkler H, Tinello D (2018) Elaboration and analysis of key figure-based approaches for the efficiency assessment of traditional and bio-inspired factory layouts. Procedia CIRP 72:732-738. https://doi.org/10.1016/j.procir.2018.03.052

13. Virnich M (1985) Selecting standard systems of production and inventory control (PIC) with the aid of utility value analysis. Toward the factory of the future. Springer Berlin Heidelberg, Berlin, Heidelberg, pp 101-107

14. Schuh G, Potente T, Fuchs S, Hausberg C (2012) Methodology for the assessment of changeability of production systems based on ERP data. Procedia CIRP 3:412-417. https://doi.org/10.1016/j.procir.2012.07.071

15. Klemke T, Nyhuis $P$ (2009) Lean changeability-evaluation and design of lean and transformable factories. Int J Econ Manag Eng. https://doi.org/10.5281/zenodo. 1327798

16. Abdi MR (2009) Layout configuration selection for reconfigurable manufacturing systems using the fuzzy AHP. Int J Manuf Technol Manag 17:149-165

17. Jonassen DH (2012) Designing for decision making. Educ Tech Research Dev 60:341-359. https://doi.org/10.1007/s1142 3-011-9230-5 
18. Bogner A, Menz W (2009) The theory-generating expert interview: epistemological interest, forms of knowledge, interaction. In: Bogner A, Littig B, Menz W (eds) Interviewing experts. Palgrave Macmillan UK, London, pp 43-80

19. Meuser M, Nagel U (2009) The Expert interview and changes in knowledge production. In: Bogner A, Littig B, Menz W (eds) Interviewing experts. Palgrave Macmillan UK, London, pp 17-42

20. Kallio H, Pietilä AM, Johnson M, Kangasniemi M (2016) Systematic methodological review: developing a framework for a qualitative semi-structured interview guide. J Adv Nurs 72:2954-2965. https://doi.org/10.1111/jan.13031

21. M. Meuser, U. Nagel (2009) The expert interview and changes in knowledge production. In: Interviewing experts. Springer, Berlin

22. ElMaraghy HA, Wiendahl H-P (2009) Changeability-an introduction. In: ElMaraghy HA (ed) Changeable and reconfigurable manufacturing systems. Springer London, London, pp 3-24

23. Böning C (2017) Quantitative, mehrdimensionale ad hoc Fabrikbewertung mittels mathematischer Modellierung von fabrikplanungsrelevanten Eigenschaften. IPH-Institut für Integrierte Produktion Hannover GmbH, Hannover
24. Heger CL (2007) Bewertung der Wandlungsfähigkeit von Fabrikobjekten. Zugl.: Hannover, Univ., Diss., 2006 \% 16, Garbsen

25. Behzadian M, Khanmohammadi Otaghsara S, Yazdani M, Ignatius J (2012) A state-of the-art survey of TOPSIS applications. Expert Syst Appl 39:13051-13069. https://doi.org/10.1016/j. eswa.2012.05.056

26. Ginevičius R, Others (2008) Normalization of quantities of various dimensions. J Bus Econ Manag 79-86

27. Zhang H, Deng Y, Chan F, Zhang X (2013) A modified multicriterion optimization genetic algorithm for order distribution in collaborative supply chain. Appl Math Model 37:7855-7864

28. Schuh G, Kampker A, Wesch-Potente C (2011) Condition based factory planning. Prod Eng Res Devel 5:89-94. https://doi. org/10.1007/s11740-010-0281-y

Publisher's Note Springer Nature remains neutral with regard to jurisdictional claims in published maps and institutional affiliations. 